\title{
Protocol Driven Peri-Procedural Anticoagulation for Left Atrial Ablation
}

\author{
Christopher Bradley ${ }^{1}$, Brian Williamson ${ }^{1}$, Julie George ${ }^{1}$, and David Haines ${ }^{1}$ \\ ${ }^{1}$ Beaumont Health System
}

August 12, 2020

\begin{abstract}
Introduction: A weight-based heparin dosing policy that adjusted for pre-procedural oral anticoagulation was implemented to reduce the likelihood of sub-therapeutic dosing during left atrial catheter ablation procedures. Our hypothesis was that initiation of the protocol would result in a greater prevalence of therapeutic Activated Clotting Time (ACT) values and decreased time to therapeutic ACT during left atrial ablation procedures. Methods: A departmental protocol was initiated for which subjects received intravenous unfractionated heparin (UFH) to achieve and maintain a goal of ACT $>300$ seconds. Initial bolus dose was adjusted for pre-procedure oral anticoagulation and weight as follows: 50 units $/ \mathrm{kg}$ for those receiving warfarin, 75 units/kg for those not anticoagulated, and $120 \mathrm{units} / \mathrm{kg}$ for those on direct oral anticoagulants. An UFH infusion was initiated at $10 \%$ of the bolus per hour. An observational study was performed on 100 consecutive left atrial ablation procedures with usual care, versus 100 with protocol guided peri-procedural care. Results: When usual care and protocol guided cohorts were compared, significant findings were limited to those on pre-procedure direct oral anticoagulant (DOAC). The initial UFH bolus increased from $97 \pm 29$ units $/ \mathrm{Kg}$ to $113 \pm 29$ units $/ \mathrm{Kg}(\mathrm{p}<0.001)$, the proportion of therapeutic ACT on first draw after heparin administration increased from $58 \%$ to $77 \%(\mathrm{p}=0.010)$, and the time to therapeutic ACT after UFH administration decreased from $37.8 \pm 19.8$ minutes to $30.2 \pm 16.4$ minutes $(\mathrm{p}=0.032)$. Conclusion: A weight-based protocol for peri-procedural UFH administration resulted in a higher proportion of therapeutic ACT values and decreased the time to therapeutic ACT for those on pre-procedure DOAC.
\end{abstract}

\author{
Abbreviations \\ ACT - Activated clotting time \\ BMI - Body mass index \\ CAD - Coronary artery disease \\ CHF - Congestive heart failure \\ DM - Diabetes Mellitus \\ DOAC - Direct oral anticoagulation \\ GFR - Glomerular filtration rate \\ HTN - Hypertension \\ LVEF - Left ventricular ejection fraction \\ RF- Radiofrequency
}

UFH - Unfractionated heparin

\section{INTRODUCTION}


Arrhythmia treatment via left atrial ablation procedures is associated with peri-procedural risk of thromboembolism at a reported rate of 0-7\%. ${ }^{1}$ To mitigate this risk, meticulous peri-procedural practices should be employed including pre-procedural imaging to rule out left atrial thrombus, vigilant sheath management, careful control of radiofrequency (RF) energy, and a strict peri-procedural anticoagulation strategy. Prior studies have demonstrated that thrombi can form on sheaths or catheters almost immediately after transseptal puncture, and peri-procedural anticoagulation via intravenous heparin can reduce this risk. ${ }^{2,3} \mathrm{~A}$ therapeutic Activated Clotting Time (ACT) goal of $>300$ seconds should be targeted, as this value has been associated with reduced thromboembolic complications, without increased bleeding complications. ${ }^{4,5}$

Contemporary anticoagulation practices during left atrial catheter ablation procedures can vary among operators, particularly in regard to the effect of patient size and peri-procedural oral anticoagulation. To reduce the likelihood of sub-therapeutic heparin dosing, a weight-based heparin dosing policy which adjusted for pre-procedural oral anticoagulation was implemented. We performed an observational quality improvement study to evaluate whether an implemented protocol would result in altered dosing practices, a greater prevalence of therapeutic ACT values, and a decrease in time to therapeutic ACT during left atrial ablation procedures compared to a retrospective cohort of similar patients.

\section{METHODS}

\section{Study Design}

After approval from our Institutional Review Board, data were collected on all consecutive patients from a single center that underwent left atrial ablation procedures performed between March $18^{\text {th }}, 2018$ and December $18^{\text {th }}, 2018$. As part of a quality improvement effort to standardize peri-procedural anticoagulation, a dosing protocol was initiated on August $9^{\text {th }}, 2018$. Outcomes before (usual care), and after protocol implementation (protocol guided) were confirmed by chart review and compared. All patients were evaluated after protocol initiation, and a retrospective comparison group (usual care) was collected on consecutive patients preceding protocol initiation. Demographic data, clinical characteristics and administered medications were recorded, as well as anticoagulation outcomes and procedural complications. Left atrial ablation was performed via transseptal access, and UFH was used for anticoagulation. If patients underwent more than one ablation within the data collection time period, only the first procedure data were included to eliminate dosing bias. During the data collection period, a total of 8 operators performed left atrial ablation procedures.

\section{Peri-Procedural Anticoagulation Protocol}

Patients underwent ablation procedures in the left atrium under general anesthesia using standard techniques. Ablation practices, pre-procedure oral anticoagulant regimen, and screening of possible drug interactions were left to the discretion of the treating physician. If used, warfarin was administered before and after the procedure without interruption. If patients were receiving DOAC pre-procedure, they were held 0-2 doses before the procedure. Bolus doses of heparin were administered prior to, or immediately after transseptal puncture, either initially as one dose, or a divided dose before and immediately after transseptal access at the operator's discretion. If the dose was divided, the combined administered dose was recorded as the bolus amount.

A departmental heparin protocol was initiated for left atrial ablation procedures based on published weightbased dosing protocols with an acceptable safety record. ${ }^{6}$ Patients received intravenous UFH with a goal to achieve and maintain an ACT $>300$ seconds. The initial prescribed bolus dose was: 50 units $/ \mathrm{kg}$ for patients receiving warfarin, 75 units $/ \mathrm{kg}$ for patients not receiving oral anticoagulation, and $120 \mathrm{units} / \mathrm{kg}$ for patients receiving DOACs. An infusion of UFH was initiated at $10 \%$ of the bolus dose per hour. ACTs were to be drawn and analyzed (Hemochron Signature Elite, Werfen, Inc., Barcelona, Spain) every 15 minutes until therapeutic levels are reached. The protocol delineated that the first ACT was to be drawn 15 minutes after heparin administration. The time to first therapeutic ACT was measured from time zero to the first documented therapeutic ACT, where time zero was denoted in two methods; (A) from initial heparin administration, and (B) from transseptal puncture. Once a therapeutic ACT was established, repeat ACTs were drawn 
a minimum of every 30 minutes. Additional bolus doses and infusion rates were at the discretion of the operator. Ablation was not to be performed until a therapeutic ACT had been obtained. The usual care cohort was not guided by an institutional protocol, thus heparin bolus, infusion, and pre-procedure oral anticoagulation regimen were left to the discretion of the operator.

After the procedure was completed, sheaths were removed and hemostasis obtained by either manual pressure after heparin reversal with protamine, or figure of eight suture placement. ${ }^{7}$ In uncomplicated cases, oral anticoagulation was restarted or continued the evening after the procedure.

\section{Study Endpoints}

The endpoints selected to assess anticoagulation success with usual care, versus a protocol guided approach were: (A) The proportion of therapeutic ACT's on first draw after heparin administration; (B) Time to therapeutic ACT after transseptal access; (C) Time to therapeutic ACT after heparin administered; (D) The proportion of therapeutic ACT during the case; (E) The proportion of supra-therapeutic ACT during the case, defined as greater than 400 seconds, and (F) The proportion of supra-therapeutic ACT's on first draw after heparin administration.

Major complication endpoints were all cause death, major thrombotic events, and major bleeding events within 30 days of the procedure. Major thrombotic events included ischemic stroke, transient ischemic attack, peripheral embolic events, and deep vein thrombosis. Major hemorrhagic events were those requiring surgical intervention, pericardial effusion requiring drainage, bleeding resulting in transfusion $>2$ units of blood, or fatal bleeding.

\section{Statistical Methods}

Cohort characteristics were compared using Pearson chi-square (or Fisher's exact test where cell counts were small) for categorical data, presented as frequencies and percentages. Continuous data, presented as means with standard deviations, were compared using analysis of variance or Wilcoxon rank sum test. Chi-square was used to compare proportion at goal on first ACT draw. T-tests were used to analyze weight bolus and time outcomes. All statistical analyses were conducted using SAS for Windows 9.4 (Cary, NC). For the analysis of therapeutic and or supra-therapeutic proportions, we accounted for variable number of ACT draws per procedure. The number of ACT draws collected ranged from 1 to 14, and consequently the number of therapeutic ACT draws was also variable. Logistic regression was utilized to analyze the average success rates between the usual care and protocol guided cohorts, taking into account the variable number of draws. The odds ratio represents the increase in odds of achieving a value $>300$ and $>400$ seconds respectively. For those without pre-procedure anticoagulation, the weight-based bolus was plotted against first ACT result. We used a censored regression analysis to account for times recorded at the Hemochron maximum value of 400 seconds, but were likely higher.

\section{RESULTS}

\section{Baseline characteristics}

There were 208 consecutive left atrial ablation cases performed. Two cases were excluded as there was a contraindication to heparin administration, with the resultant use of an alternate intravenous anticoagulant. Another 6 procedures were excluded, as 3 patients had repeat ablation for which procedures existed in both the usual care and protocol guided cohorts. After exclusions, 200 patients were analyzed, 100 in each cohort. There was no difference between the usual care and protocol guided cohorts in regard to demographics, antiplatelet medications prior to procedure, medical comorbidities, mean glomerular filtration rate (GFR), and mean left ventricular ejection fraction (Table 1).

Oral anticoagulant use prior to procedure was similar between the two cohorts. There were 78 and 77 patients on DOACs in the usual care and protocol guided arms respectively, with an increase in apixaban (from 49 to 54 patients), and decrease in rivaroxaban (from 27 to 21 patients) use in the protocol guided cohort. In the usual care cohort there were 12 patients on uninterrupted warfarin, and 10 patients on no oral anticoagulant 
prior to procedure. This compared similarly to the protocol guided cohort as there were 11 patients on uninterrupted warfarin, and 12 patients on no oral anticoagulant prior to procedure. The usual care cohort included 15 of the 78 patients (19.2\%) patients for which their direct oral anticoagulant was held for more than 2 doses prior to procedure, all of which were on twice daily dosed medications, and only two patients were interrupted for more than 3 doses. This compared to 7 out of $77(9.1 \%)$ in the protocol guided cohort for which 6 patients were on twice daily dosed medications, and only one patient was interrupted for more than 3 doses.

\section{Procedural Characteristics}

The majority of left atrial ablation procedures evaluated were for treatment of atrial fibrillation. Of the 100 patients in the usual care ablation cohort, 85 received treatment for atrial fibrillation, 4 for left sided accessory pathway, and 12 for left atrial tachycardia. Of the 100 patients in the protocol guided ablation cohort, 93 received treatment for atrial fibrillation, 5 for left sided accessory pathway, and 2 for left atrial tachycardia.

The average number of ACT's drawn per procedure were similar between each cohort, as an average of $5.9 \pm$ 2.4 were drawn in the usual care cohort, as opposed to $5.7 \pm 2.4$ in the protocol guided cohort. The average time to ACT after initial heparin bolus was $25 \pm 13$ minutes in the usual care, and $25 \pm 13$ minutes in the protocol guided cohorts. There was a difference in timing of heparin administered with regard to transseptal puncture, as heparin was administered prior to transseptal puncture in the usual care cohort at a lower rate $(77 \%)$, compared the protocol guided arm (94\%). The initial heparin bolus was split (before and after transseptal puncture) in a high proportion in the usual care cohort (30\%), when compared to the protocol guided cohort (13\%).

\section{Outcomes}

After the peri-procedural heparin protocol was initiated, the initial weight-based bolus of heparin increased from $97 \pm 29$ units $/ \mathrm{Kg}$ to $113 \pm 29$ units $/ \mathrm{Kg}(\mathrm{p}<0.001)$. ACT outcomes are shown in Figure 1. Therapeutic values on first draw increased from $60 \%$ in the usual care cohort, to $77 \%$ in the protocol guided cohort (Figure 1A, $\mathrm{p}=0.010$ ). And there was not a significant increase in supra-therapeutic values on first ACT value after heparin administration (Figure $1 \mathrm{~B}, 12 \%$ versus $20 \%, \mathrm{p}=0.123$ ). The total proportion of ACT values in therapeutic range during the procedure increased from $79 \%$ to $86 \%$ with protocol guidance (Figure 1C, OR 1.68, 95\% CI $1.24-2.28, \mathrm{p}=0.001)$. Supra-therapeutic proportions also did not significantly increase between the usual care and protocol guided cohorts (21\% versus $25 \%$, Figure 1D, OR 1.26, 95\% CI $0.89-$ $1.78, \mathrm{p}=0.198)$.

Time to therapeutic ACT decreased after initiation of the heparin protocol (Figure 2). Time from heparin administration to therapeutic ACT deceased from $37.3 \pm 18.8$ minutes with usual care, to $31.1 \pm 16.4$ minutes in the protocol guided cohort $(\mathrm{p}=0.034)$. Similarly, time from transspetal access to therapeutic ACT decreased from $30.2 \pm 22.3$ min with usual care to $19.8 \pm 17.3$ in the protocol guided cohort $(\mathrm{p}=0.001)$.

There was only one major bleeding or thromboembolic event in either cohort, which was a pseudoaneurysm formation with concomitant ipsilateral common femoral vein thrombus identified in the protocol guided cohort.

\section{Outcomes and Pre-procedure Anticoagulation Regimen}

When outcomes were assessed based upon pre-procedure oral anticoagulant regimen, patients on DOAC demonstrated significant outcome differences between cohorts. For those on DOAC, the initial weight-based bolus of heparin increased from $99 \pm 25$ units $/ \mathrm{Kg}$ to $118 \pm 23$ units $/ \mathrm{Kg}(\mathrm{p}<0.001)$. Therapeutic values on first draw increased from $58 \%$ to $77 \%$ with protocol guidance $(\mathrm{p}=0.010)$. However, supra-therapeutic values on first ACT draw did significantly increase from $6 \%$ with usual care, to $18 \%$ in the protocol guided cohort ( $\mathrm{p}=0.026$ ). Therapeutic proportions increased from $78 \%$ with usual care to $86 \%$ in the protocol guided cohort (OR $1.89,95 \%$ CI $1.36-2.64, \mathrm{p}=<0.001$ ). Supra-therapeutic proportions did not significantly increase from $18 \%$ with usual care versus $23 \%$ in the protocol guided cohort (OR 1.44, 95\% CI $0.98-2.13, \mathrm{p}=0.067$ ). Time 
to therapeutic ACT, when measured after heparin administration, deceased from $38 \pm 20$ minutes to $30 \pm 17$ minutes $(\mathrm{p}=0.032)$, and time to therapeutic ACT after transseptal access decreased from $32 \pm 23$ with usual care to $19 \pm 17$ minutes with protocol guidance $(\mathrm{p}=0.001)$.

For those patients receiving pre-procedure warfarin, the dose of heparin administered in the protocol guided cohort was higher $(71 \pm 39$ units $/ \mathrm{Kg})$, than the recommended protocol dose (50 units/Kg) and compared similarly to the usual cohort $70 \pm 36$ units $/ \mathrm{Kg}$. As a result, the proportion of therapeutic ACT values on first draw (100\%), and the proportion of supra-therapeutic (45.5\%) values were higher than any of the other preprocedure oral anticoagulant strategies. However lower patient numbers in this subgroup limited meaningful analysis.

For the patients that received no oral anticoagulant before the procedure, the average bolus dose in the protocol guided cohort was higher $(116 \pm 28$ units $/ \mathrm{Kg})$ than that recommended by the protocol $(75 \mathrm{U} / \mathrm{Kg})$ and compared similarly to the usual cohort $112 \pm 10$ units/Kg. Despite this observation, the proportions of therapeutic ACT values on first draw remained lower (58\%) than the overall protocol guided cohort findings $(77 \%)$, and there was no significant change between the usual care and protocol guided cohorts. This suggests that patients not anticoagulated pre-procedure may be under dosed by the protocol design. To gain insight into the proper bolus dosing for this subgroup, we plotted the weight-based heparin bolus dose versus the first ACT value after administration, and performed linear regression analysis (Figure 4). As a result, a weight-based bolus of at least $120 \mathrm{U} / \mathrm{Kg}$ may be a more appropriate for those on no pre-procedure oral anticoagulant, however a prospective validation of such a weight-based dose is required.

\section{DISCUSSION}

A protocol guided approach which accounted for pre-procedure oral anticoagulation and weight resulted in a higher administered dose, increased proportion of therapeutic ACT values, and a decreased time to therapeutic ACT. The findings were driven by a large number of those on pre-procedure DOAC, which reflects contemporary practice. The complexity of left atrial ablation procedures requires attention to multiple variables, and the standardization of peri-procedural anticoagulation by means of protocol guidance may result in a reduction in untoward outcomes.

Much attention and investigation has been directed towards the optimal choice of pre-procedural oral anticoagulation, and whether to proceed with an interrupted versus uninterrupted strategy. For those randomized trials which investigated uninterrupted pre-procedure oral anticoagulant administration during AF ablation, a fixed dose based on sex or weight was given. These trials did not address both body weight and prior oral anticoagulation strategy while dosing UFH to achieve a therapeutic ACT. ${ }^{8-12}$ However, intravenous unfractionated heparin pharmacokinetics should be considered, as they are dose and weight dependent. The half-lives of 25, 100, and $400 \mathrm{U} / \mathrm{kg} \mathrm{UFH}$ are 30,60, and $150 \mathrm{~min}$, respectively. At lower doses, the infused heparin binds directly to macrophages and endothelial cells, where it gets depolymerized. At higher doses, this mechanism becomes saturated. ${ }^{13}$ Continuous infusion after bolus may allow for a steady state UFH plasma level, and lead to increased proportion of therapeutic ACT levels. ${ }^{14}$

In addition to weight, pre-procedure OAC should be considered given the preponderance of data indicates that ACT response differs when warfarin is compared to DOAC therapies. ${ }^{4,15}$ Pre-procedure DOAC results in larger required weight-based peri-procedural UFH bolus to achieve a desired ACT of $>300$ seconds. ${ }^{15,16}$ Additionally, body weight has been suggested as a predictor of UFH requirement for those who are on pre-procedure rivaroxaban, during left atrial ablation. ${ }^{17}$

To minimize the burden on the operator and decrease the proportion of sub-therapeutic ACT values, an institutional protocol was initiated with previously validated safety outcomes. ${ }^{6}$ We leveraged the temporal initiation of the peri-procedural anticoagulation protocol to evaluate whether it had an effect on dosing practices, and ACT outcomes. A peri-procedural anticoagulation protocol resulted in a higher dose of heparin administered as the initial bolus and improved ACT outcomes, primarily driven by dosage changes in patients receiving a pre-procedure DOAC. Those on DOAC (particularly the Factor Xa inhibitors) were the majority of patients evaluated, reflecting contemporary anticoagulation practices. Heparin under-dosing in patients 
receiving DOACs may have been influenced by the omnipresent risk of bleeding complications and the lack of an easily obtained reversal agent for the factor Xa inhibitors. The initiation of a peri-procedural anticoagulation protocol may have empowered the operators in the study to administer larger bolus doses of UFH, which resulted in earlier and increased proportion of therapeutic ACT values. Sub-therapeutic ACT values have been correlated to silent ischemic events identified on MRI, ${ }^{5}$ which further highlights the value of peri-procedural anticoagulation strategies to balance safety with efficacy.

The small numbers of patients on warfarin limit meaningful conclusion. Patients receiving warfarin were administered larger bolus dose than initially recommended by our protocol, resulting in both a high number of therapeutic and supra-therapeutic ACT values. Patients receiving no pre-procedure oral anticoagulation received larger doses than the protocol recommended, yet had lower rates of therapeutic ACTs. The employed protocol mirrored weight based recommendations based on previous data with adequate safety profile, for which those on no pre procedure anticoagulation received a lower heparin bolus than those on DOAC. Linear regression analysis of these patients revealed that a dose of at least $120 \mathrm{U} / \mathrm{Kg}$ was likely required to achieve an ACT of 300 seconds in half of the patients, however prospective validation is required before delivering a definitive weight-based recommendation in those without pre-procedure oral anticoagulant.

Limitations

This study has several limitations. The observational design did not allow for control of several important variables, in particular the heparin dose received, which was at physician discretion. In the protocol guided arm, there was operator adherence to the proposed bolus in those on pre-procedure DOAC. However, in the protocol guided arm the average bolus dose administered in those who received pre-procedure warfarin and no anticoagulant were greater than the protocol recommended bolus dose. There was an absolute increase of $17 \%$ in the proportion of heparin given prior to transseptal puncture in the protocol guided cohort, and an absolute decrease of $17 \%$ in the proportion of heparin bolus which were split before and after transseptal puncture. The change in bolus modality and timing may have been an additional effect of the protocol on physician practice, and certainly affected the reduction in time from transseptal to therapeutic ACT. However, a reduction in time to therapeutic ACT persisted when measured from time of heparin administration Anticoagulant therapy and dosing was prescribed at the operator's discretion, and not prospectively assigned, possibly introducing selection bias. Additionally, there is assumption that patients were adherent to preoperative instructions for managing their anticoagulant therapy. Finally, the ACT machine did not provide an absolute value for values over 400 , rather a truncated value.

\section{CONCLUSION}

A weight-based protocol for peri-procedural heparin administration which accounted for oral anticoagulation resulted in a higher proportion of therapeutic ACT, higher administered bolus dose, and decreased the time to therapeutic ACT for those on pre-procedure DOAC. A weight-base bolus dosing protocol may serve as a contemporary template for future peri-procedural anticoagulation strategies during left atrial ablation.

Acknowledgements: The authors would like to thank Divyashree Varma, MD, Ilana Kutinsky, DO, K. Ching Man, DO, David Nori, MD, Salim Ahmed, MD, Hazim Al-Ameri, MD, and Nathan Foster, MD for their procedural expertise and willingness to participate during protocol initiation. Additionally, we would like to acknowledge Shirley Qu for help in assembling the database.

\section{REFERENCES}

1. Calkins H, Hindricks G, Cappato R, et al. 2017 HRS/EHRA/ECAS/APHRS/SOLAECE expert consensus statement on catheter and surgical ablation of atrial fibrillation. Hear Rhythm . Published online 2017. doi:10.1016/j.hrthm.2017.05.012

2. Asbach S, Biermann J, Bode C, Faber TS. Early heparin administration reduces risk for left atrial thrombus formation during atrial fibrillation ablation procedures. Cardiol Res Pract . Published online 2011. doi:10.4061/2011/615087 
3. Bruce CJ, Friedman PA, Narayan O, et al. Early heparinization decreases the incidence of left atrial thrombi detected by intracardiac echocardiography during radiofrequency ablation for atrial fibrillation. $J$ Interv Card Electrophysiol . Published online 2008. doi:10.1007/s10840-008-9270-x

4. Briceno DF, Villablanca PA, Lupercio F, et al. Clinical impact of heparin kinetics during catheter ablation of atrial fibrillation: Meta-analysis and meta-regression. J Cardiovasc Electrophysiol . Published online 2016. doi:10.1111/jce.12975

5. Di Biase L, Gaita F, Toso E, et al. Does periprocedural anticoagulation management of atrial fibrillation affect the prevalence of silent thromboembolic lesion detected by diffusion cerebral magnetic resonance imaging in patients undergoing radiofrequency atrial fibrillation ablation with o. Hear Rhythm . Published online 2014. doi:10.1016/j.hrthm.2014.03.003

6. Armbruster HL, Lindsley JP, Moranville MP, et al. Safety of Novel Oral Anticoagulants Compared With Uninterrupted Warfarin for Catheter Ablation of Atrial Fibrillation. Ann Pharmacother. Published online 2015. doi:10.1177/1060028014563950

7. Lakshmanadoss U, Wong WS, Kutinsky I, Khalid MR, Williamson B, Haines DE. Figure-of-eight suture for venous hemostasis in fully anticoagulated patients after atrial fibrillation catheter ablation. Indian Pacing Electrophysiol J . Published online 2017. doi:10.1016/j.ipej.2017.02.003

8. Di Biase L, Burkhardt JD, Santangeli P, et al. Periprocedural stroke and bleeding complications in patients undergoing catheter ablation of atrial fibrillation with different anticoagulation nagement results from the role of coumadin in preventing thromboembolism in atrial fibrillation (AF) patients u. Circulation . Published online 2014. doi:10.1161/CIRCULATIONAHA.113.006426

9. Cappato R, Marchlinski FE, Hohnloser SH, et al. Uninterrupted rivaroxaban vs. uninterrupted vitamin $\mathrm{K}$ antagonists for catheter ablation in non-valvular atrial fibrillation. Eur Heart $J$. Published online 2015. doi:10.1093/eurheartj/ehv177

10. Calkins H, Willems S, Gerstenfeld EP, et al. Uninterrupted dabigatran versus warfarin for ablation in atrial fibrillation. $N$ Engl $J$ Med . Published online 2017. doi:10.1056/NEJMoa1701005

11. Kuwahara T, Abe M, Yamaki M, et al. Apixaban versus Warfarin for the Prevention of Periprocedural Cerebral Thromboembolism in Atrial Fibrillation Ablation: Multicenter Prospective Randomized Study. $J$ Cardiovasc Electrophysiol . Published online 2016. doi:10.1111/jce.12928

12. Hohnloser SH, Camm J, Cappato R, et al. Uninterrupted edoxaban vs. Vitamin K antagonists for ablation of atrial fibrillation: The ELIMINATE-AF trial. Eur Heart $J$. Published online 2019. doi:10.1093/eurheartj/ehz190

13. Hirsh J, Anand SS, Halperin JL, Fuster V. Guide to anticoagulant therapy: Heparin: A statement for healthcare professionals from the American Heart Association. Circulation . Published online 2001. doi:10.1161/01.CIR.103.24.2994

14. Kim TS, Kim SH, Kim BK, et al. Randomized Comparison of Continuous Versus Intermittent Heparin Infusion During Catheter Ablation of Atrial Fibrillation. JACC Clin Electrophysiol . Published online 2016. doi:10.1016/j.jacep.2015.11.004

15. Konduru S V., Cheema AA, Jones P, Li Y, Ramza B, Wimmer AP. Differences in intraprocedural ACTs with standardized heparin dosing during catheter ablation for atrial fibrillation in patients treated with dabigatran vs. patients on uninterrupted warfarin. J Interv Card Electrophysiol . Published online 2012. doi:10.1007/s10840-012-9719-9

16. Payne JE, Koerber SM, Bickel T, Ghadban R, Flaker G, Gautam S. Higher initial weight-based heparin dosing is required with direct oral anticoagulants during catheter ablation for atrial fibrillation. J Interv Card Electrophysiol . Published online 2019. doi:10.1007/s10840-019-00579-y 
17. Enriquez AD, Churchill T, Gautam S, et al. Determinants of Heparin Dosing and Complications in Patients Undergoing Left Atrial Ablation on Uninterrupted Rivaroxaban. PACE - Pacing Clin Electrophysiol . Published online 2017. doi:10.1111/pace.13013

\section{FIGURE LEGEND}

Figure 1 - Demonstrated are the ACT outcomes comparing the usual care, to the protocol guided cohort. Aggregate data includes all patients regardless of pre-procedure oral anticoagulant. Those on pre-procedure DOAC, warfarin, and no oral anticoagulant (None) are also shown. Panel A shows the proportion (\%) of ACT $>300$ seconds on first draw after the heparin bolus was administered. Panel B shows the proportion (\%) of ACT $>400$ seconds on first draw after the heparin bolus was administered. Panel C shows the total proportion (\%) of ACT values > 300 seconds during the procedure. Panel D shows the total proportion (\%) of $\mathrm{ACT}$ values $>400$ seconds during the procedure.

Figure 2 - Demonstrated are the time outcomes to achieved an ACT $>300$ seconds, comparing the usual care, to the protocol guided cohort. Aggregate data includes all patients regardless of pre-procedure oral anticoagulant. Those on pre-procedure DOAC, warfarin, and no oral anticoagulant (None) are also shown. Error bars indicate standard error. Panel A shows the time to an ACT $>300$ seconds when measured after Heparin bolus administered. Panel B shows the time to an ACT > 300 seconds when measured after the transseptal puncture was performed.

Figure 3 - Demonstrated are average weight-based bolus (units/Kg), comparing the usual care, to the protocol guided cohort. Aggregate data includes all patients regardless of pre-procedure oral anticoagulant. Those on pre-procedure DOAC, warfarin, and no oral anticoagulant (None) are also shown. Error bars indicate standard error.

Figure 4 - Data from all patients receiving no pre-procedure oral anticoagulant are presented, regardless of cohort designation. The weight-based heparin bolus administered is plotted against the first ACT value after heparin administration. Blue line represents linear regression $(\mathrm{p}=0.011)$, with a goodness of fit $\mathrm{R}^{2}$ value of 0.297 . 
Figure 1, A-D

A First ACT $\geq 300$ Seconds

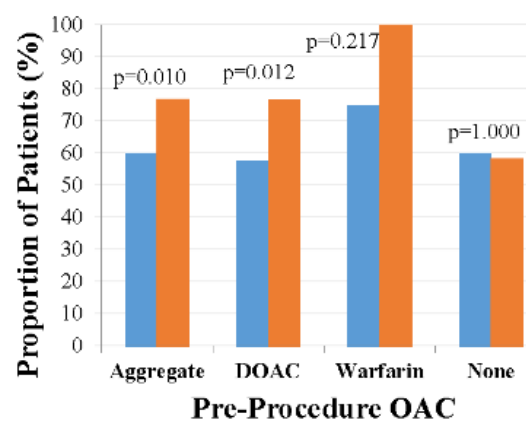

n Usual Care $\quad$ Protocol Guided

C Proportion of ACT $\geq 300$ Seconds

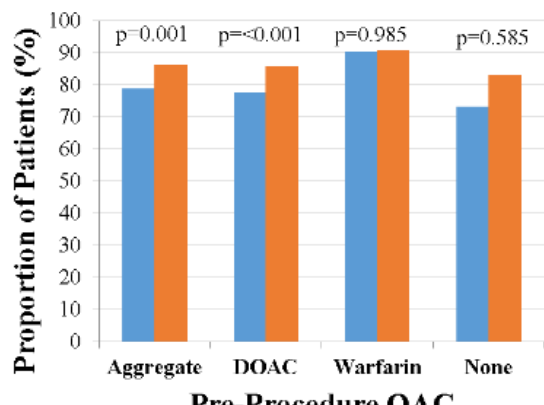

Pre-Procedure OAC

n Usual Care $\quad$ Protocol Guided

\section{B First ACT $>400$ Seconds}

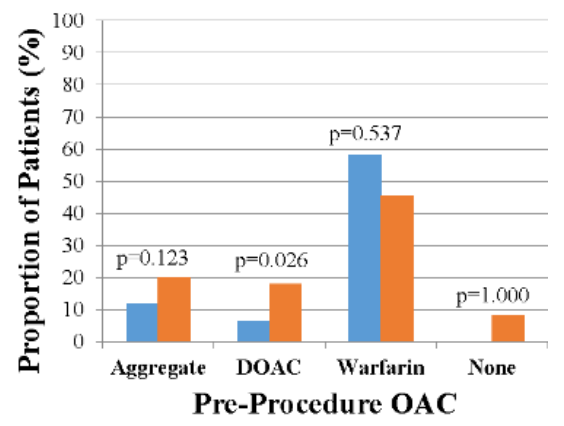

n Usual Care $\quad$ Protocol Guided

D Proportion of ACT $>400$ Seconds

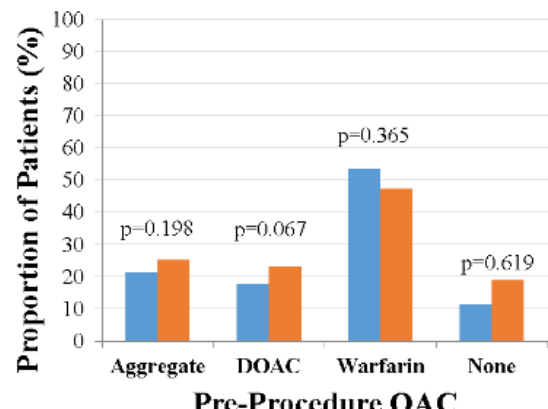

E Usual Care $\quad$ Protocol Guided

Figure 2, A-B
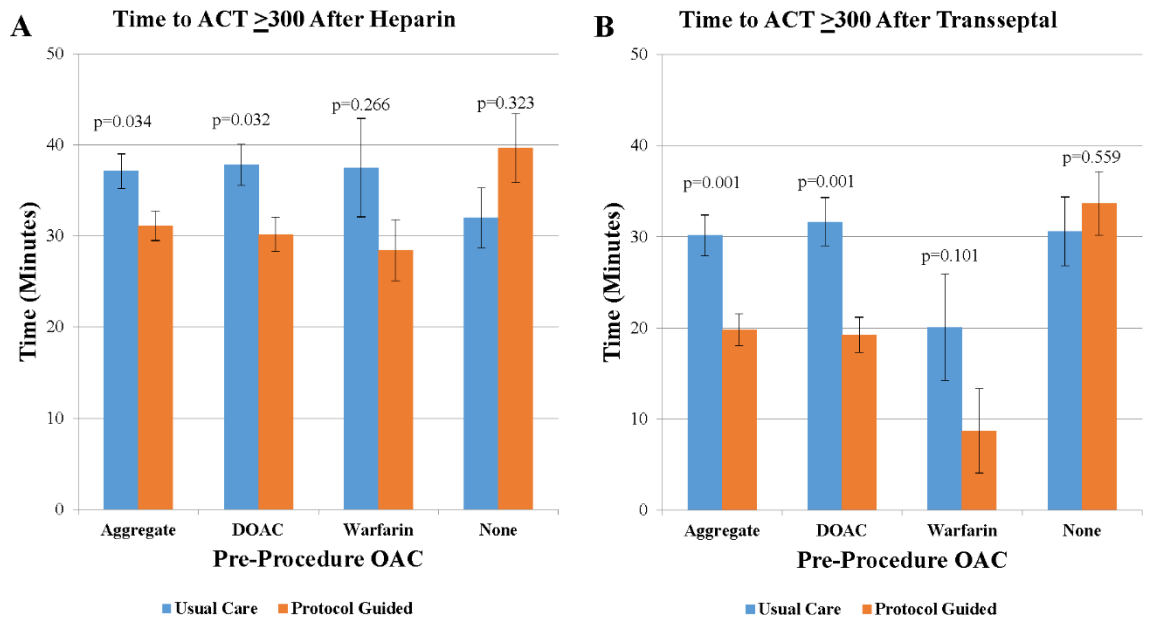
Figure 3

Weight Based Bolus Dose

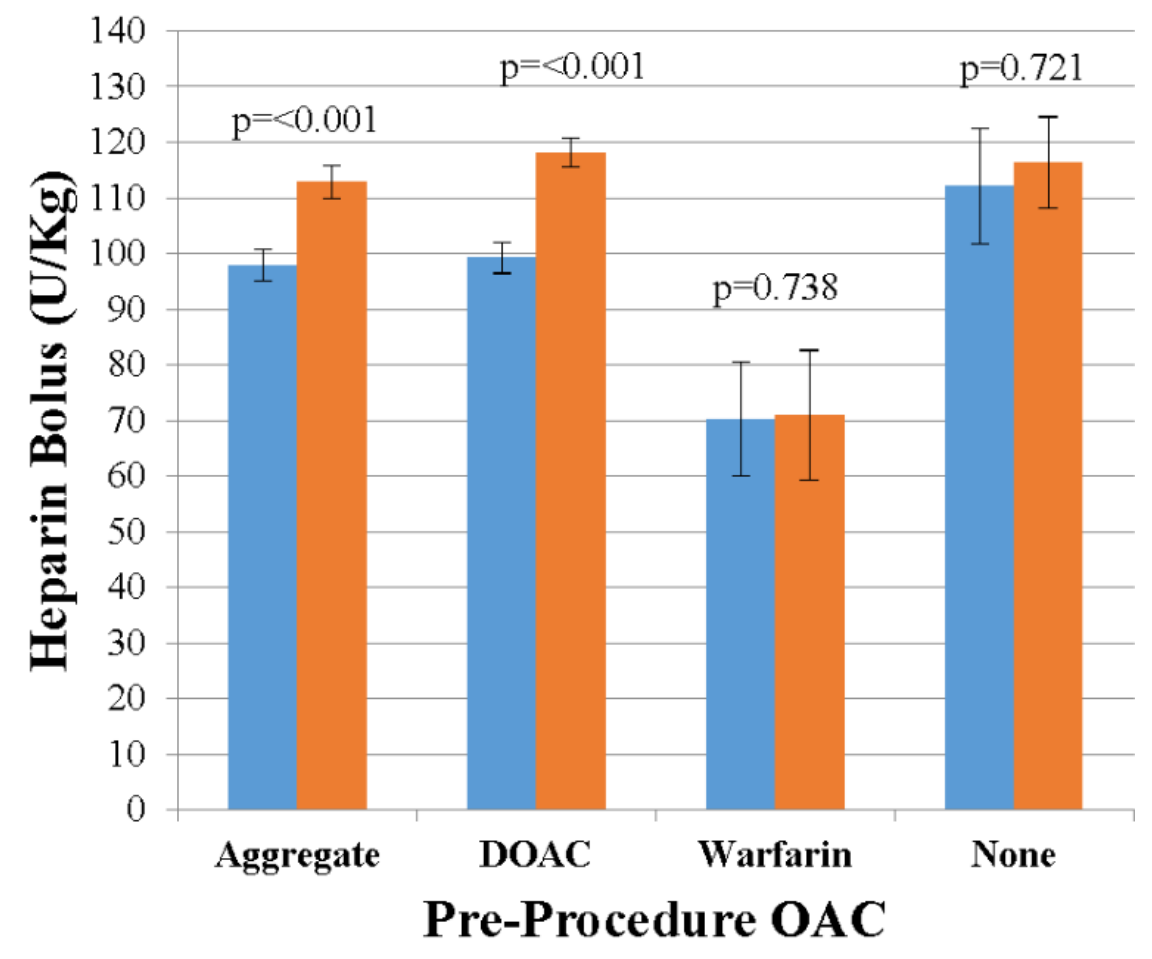

- Usual Care Protocol Guided 


\section{Figure 4}

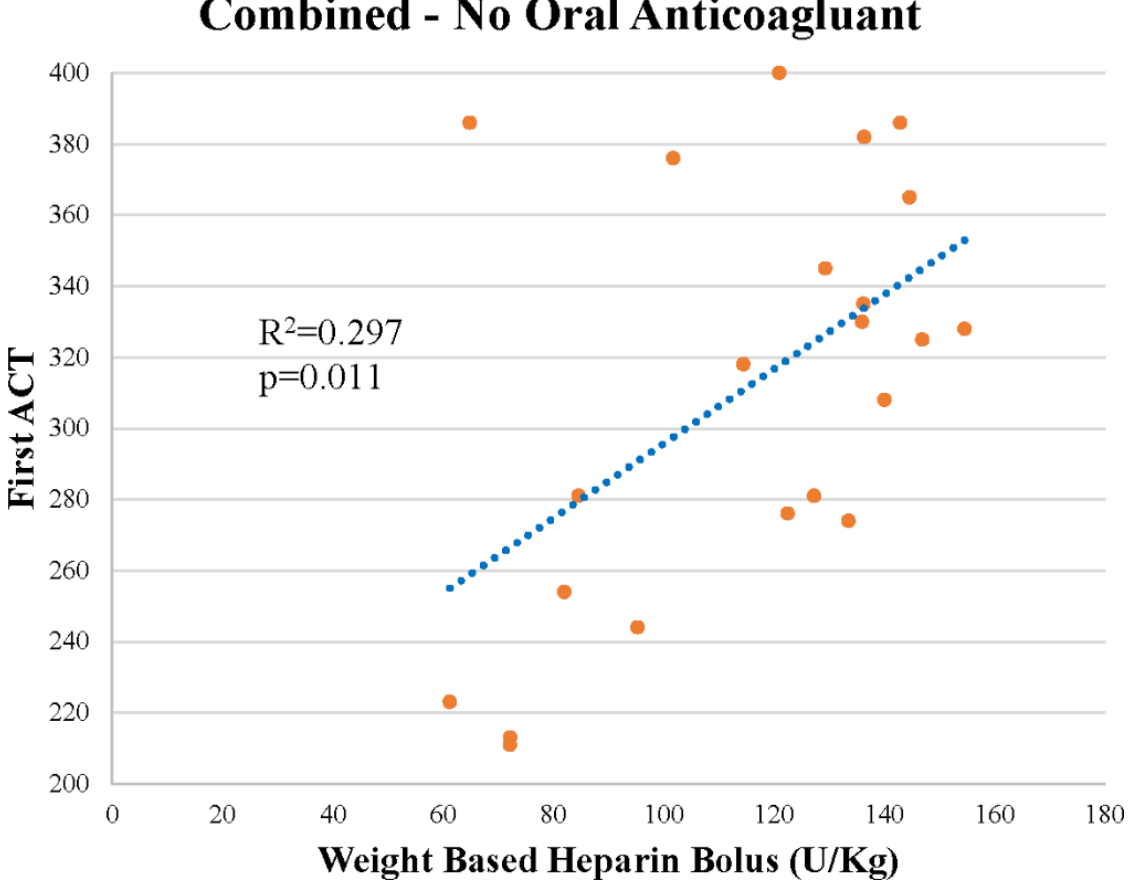

\begin{tabular}{llll}
\hline Table $\mathbf{1}$ & Table $\mathbf{1}$ & Table $\mathbf{1}$ & Table $\mathbf{1}$ \\
\hline & Usual Care $\mathbf{N = \mathbf { 1 0 0 }}$ & $\begin{array}{l}\text { Protocol Guided } \\
\mathbf{N}=\mathbf{1 0 0}\end{array}$ & P value \\
Age & $62.0 \pm 11.2$ & $59.5 \pm 10.6$ & 0.122 \\
Race, n (\%) & & $91(91)$ & \\
Caucasian (\%) & $89(89)$ & $4(4)$ & \\
Black (\%) & $5(5)$ & $1(1)$ & \\
Asian (\%) & $2(2)$ & $4(4)$ & \\
Other (\%) & $4(4)$ & $69(69)$ & 0.345 \\
Male, n (\%) & $75(75)$ & $31.3 \pm 5.9$ & 0.590 \\
BMI (kg/m $)$ & $31.0 \pm 5.7$ & $42(42)$ & 1.0 \\
Antiplatelet, $\mathbf{n}(\mathbf{\%})$ & & $4(4)$ & 1.0 \\
Aspirin & $42(42)$ & & \\
Clopidogrel & $4(4)$ & $10(10)$ & 0.103 \\
Comorbidities, $\mathbf{n}$ & & $13(13)$ & 0.547 \\
(\%) & & $12(12)$ & 0.171 \\
CHF & $18(18)$ & $5(5)$ & 0.552 \\
DM & $16(16)$ & $15(15)$ & 0.352 \\
Stroke & $19(19)$ & $66(66)$ & 0.881 \\
PAD & $7(7)$ & $80 \pm 16$ & 0.559 \\
CAD & $20(20)$ & & \\
HTN & $67(67)$ & $58 \pm 9$ & 0.210 \\
GFR & $81 \pm 15$ & &
\end{tabular}




\section{APPENDIX}

Data sources

A Beaumont Health registry was established to calculate all demographics, clinical characteristics, comorbid conditions, pertinent medications, and outcomes of patients who underwent left atrial ablation procedures. The data from the registry was abstracted through automated reports generated through a multi-platform database query tool from Beaumont Health's electronic medical record. Demographics abstracted from the registry for the purpose of this study were age, sex, race, and weight. Comorbid conditions abstracted were hypertension, diabetes mellitus, coronary artery disease, peripheral artery disease, stroke/TIA history, and congestive heart failure. Laboratory values abstracted were the peri-procedural race based GFR. The periprocedural medication administration record for each patient was abstracted from the registry and compared with the antiplatelet medications aspirin, clopidogrel, ticagrelor, prasugrel, and dipyridamole. During the pre-procedure history and physical, the duration of held oral anticoagulant doses was documented and collected in the registry.

Data review and collected was performed by a physician trained to abstract the needed data into SharePoint. Heparin doses and administration times were obtained by the medical record by review of the anesthesia procedure log. ACT values and result times were documented by the electrophysiology laboratory staff, and reviewed in the medical record. Left ventricular ejection fraction was abstracted from the electronic medical record after review of the most recent echocardiogram. All occurrences of major bleeding or thrombotic events were reviewed and adjudicated by a Board Certified Cardiologist.

A

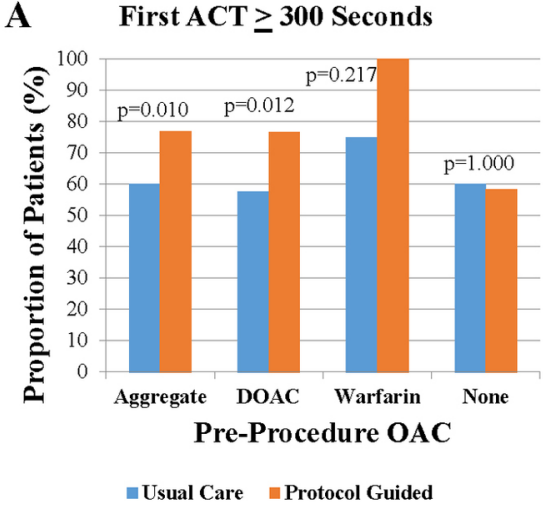

C Proportion of ACT $\geq 300$ Seconds

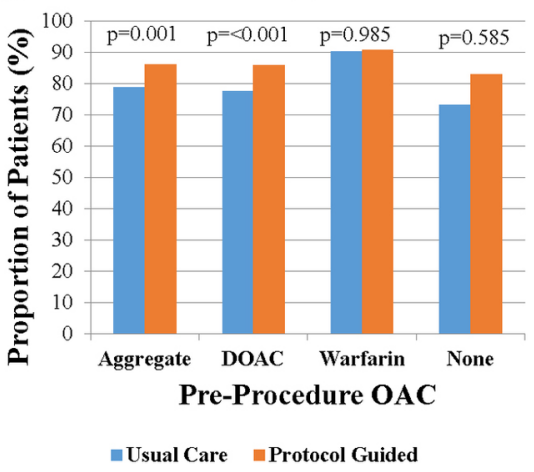

B First ACT $>400$ Seconds

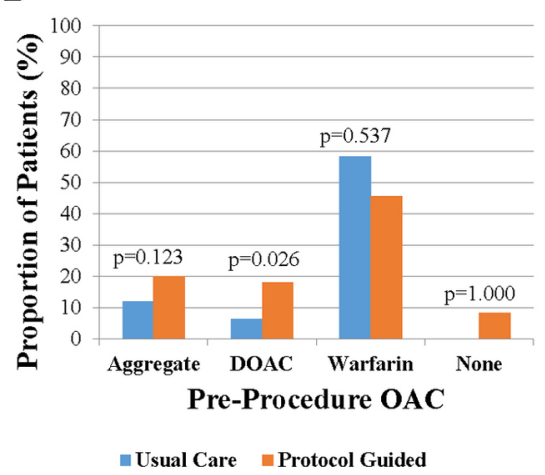

D Proportion of ACT $>\mathbf{4 0 0}$ Seconds

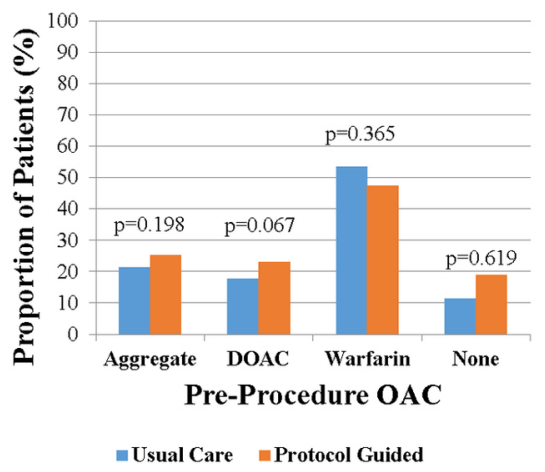



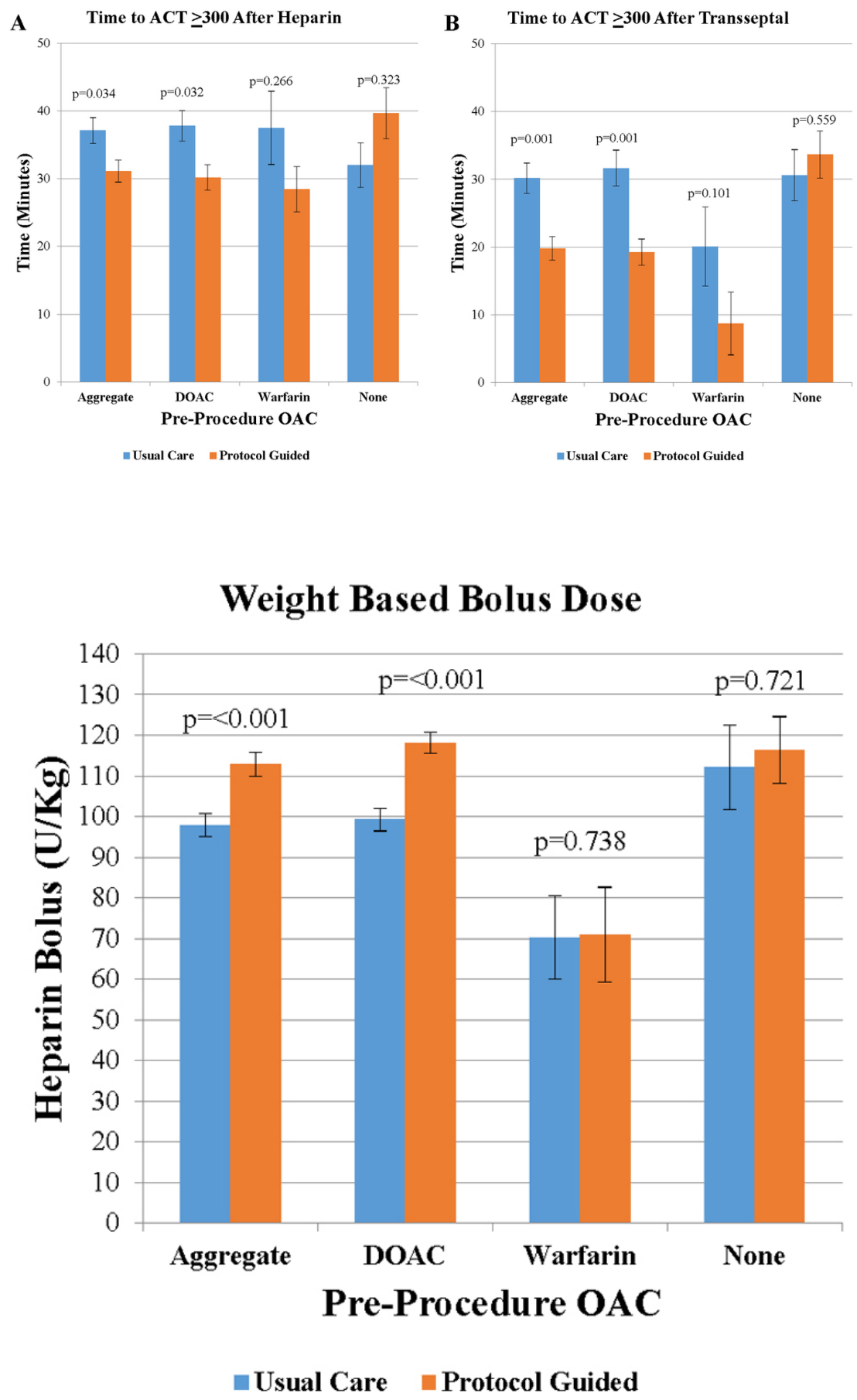


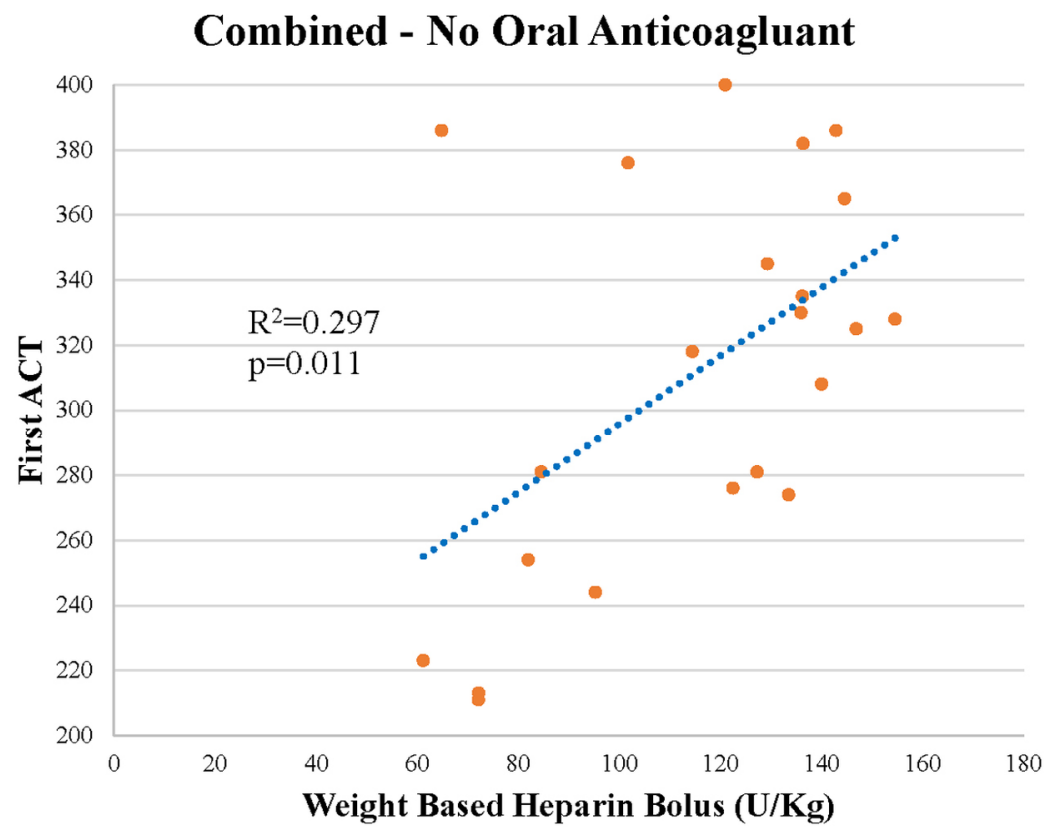

\section{Hosted file}

Table 1.docx available at https://authorea.com/users/350273/articles/475148-protocol-drivenperi-procedural-anticoagulation-for-left-atrial-ablation 